\title{
The Story of the Fibrin(ogen) $\alpha C$-Domains: Evolution of Our View on Their Structure and Interactions
}

\author{
Leonid Medved $^{1}$ John W. Weisel ${ }^{2}$ \\ ${ }^{1}$ Center for Vascular and Inflammatory Diseases and the Department \\ of Biochemistry and Molecular Biology, University of Maryland \\ School of Medicine, Baltimore, Maryland, United States \\ 2 Department of Cell and Developmental Biology, Perelman School of \\ Medicine at the University of Pennsylvania, Philadelphia, \\ Pennsylvania, United States
}

Thromb Haemost 2022;122:1265-1278.

\begin{abstract}
Address for correspondence Leonid Medved, PhD, University of Maryland School of Medicine, Center for Vascular and Inflammatory Diseases, 800 West Baltimore Street, Baltimore, MD 21201, United States (e-mail: Lmedved@som.umaryland.edu).
\end{abstract}

\begin{abstract}
Although much has been established concerning the overall structure and function of fibrinogen, much less has been known about its two $\alpha \mathrm{C}$ regions, each consisting of an $\alpha C$-connector and an $\alpha \mathrm{C}$-domain, but new information has been accumulating. This review summarizes the state of our current knowledge of the structure and interactions of fibrinogen's $\alpha C$ regions. A series of studies with isolated $\alpha C$ regions and their fragments demonstrated that the $\alpha \mathrm{C}$-domain forms compact ordered structures consisting of $\mathrm{N}$ - and C-terminal subdomains including $\beta$ sheets and suggested that the $\alpha \mathrm{C}$-connector has a poly(L-proline) type II structure. Functionally, the $\alpha \mathrm{C}$-domains interact intramolecularly with each other and with the central region of the molecule, first demonstrated by electron microscopy and then quantified by optical trap force spectroscopy. Upon conversion of fibrinogen into fibrin, the $\alpha \mathrm{C}$-domains switch from intra- to intermolecular interactions to form ordered $\alpha C$ polymers. The formation of $\alpha \mathrm{C}$ polymers occurs mainly through the homophilic interaction between the $\mathrm{N}$-terminal subdomains; interaction between the C-terminal subdomains and the $\alpha \mathrm{C}$-connectors

Keywords

- fibrinogen

- fibrin

- fibrin assembly

- fibrinogen $\alpha \mathrm{C}-$ domains

- $\alpha C$ polymers also contributes to this process. Considerable evidence supports the idea that the $\alpha \mathrm{C}$ regions accelerate fibrin polymerization and affect the final structure of fibrin clots. The interactions between $\alpha \mathrm{C}-$ regions are important for the mechanical properties of clots, increasing their stiffness and extensibility. Conversion of fibrinogen into fibrin results in exposure of multiple binding sites in its $\alpha C$ regions, providing interaction of fibrin with different proteins and cell types during hemostasis and wound healing. This heretofore mysterious part of the fibrinogen molecule is finally giving up its secrets.
\end{abstract}

\section{Introduction}

Fibrinogen is a polyfunctional plasma protein that plays an important role in hemostasis, thrombosis, wound healing, inflammation, angiogenesis, and other important physiolog- ical and pathological processes. Fibrinogen's major function in hemostasis is to form blood clots that seal damaged vasculature to prevent blood loss. Activation of the blood coagulation cascade after vascular injury results in generation of the active enzyme thrombin. This enzyme removes received

September 16, 2021

accepted after revision

December 9, 2021

published online

February 8, 2022 (c) 2022. Thieme. All rights reserved. Georg Thieme Verlag KG,

Rüdigerstraße 14,

70469 Stuttgart, Germany
DOI https://doi.org/

10.1055/a-1719-5584.

ISSN 0340-6245. 
from fibrinogen two pairs of fibrinopeptides, fibrinopeptides $\mathrm{A}(\mathrm{FpA})$ and fibrinopeptides $\mathrm{B}(\mathrm{FpB})$, converting monomeric fibrinogen into fibrin. Monomeric fibrin spontaneously polymerizes to form fibrin polymers representing the structural and mechanical basis of blood clots. Fibrinogen is rather inert in the circulation; however, it becomes highly reactive after conversion to monomeric and then polymeric fibrin. Namely, fibrin polymers in blood clots deposited on the wound activate the fibrinolytic system and serve as a provisional matrix on which different cell types adhere, migrate, and proliferate during wound healing to eventually replace blood clots with natural tissues. Such a polyfunctional character of fibrin(ogen) is connected with its multidomain structure, in which individual domains containing various binding sites are involved in numerous interactions, thereby providing the means for the participation of fibrinogen in the abovementioned processes.

The crystal structure of about two-thirds of the fibrinogen molecule, including multiple domains, has been established by X-ray analysis, and functional roles of these domains, especially those containing major polymerization sites, have been well characterized. However, the structure of the remaining portions of this molecule, which account for about one-fourth of the molecule and are often called the $\alpha \mathrm{C}$ domains, ${ }^{1}$ has not been resolved. There is a pair of such domains in the fibrinogen molecule, each of them is formed by the C-terminal portion of fibrinogen's A $\alpha$ chain. These domains play an important role in fibrin assembly, fibrinolysis, angiogenesis, and other physiological and pathological processes. Their pathophysiological role has been recently reviewed by Soria and collaborators. ${ }^{2}$ We reviewed the structure and function of the $\alpha \mathrm{C}$-domains two decades ago. ${ }^{1}$ Since then, numerous studies have been performed to clarify their structure and function. In the present review, we summarize more recent studies performed mainly in our two laboratories on the structure and interactions of the $\alpha C$ domains that shed light on the molecular mechanisms of their actions.

\section{Fibrinogen Structure}

The fibrinogen molecule is a chemical dimer consisting of two identical subunits, each of which is formed by three nonidentical polypeptide chains, $A \alpha, B \beta$, and $\gamma$, linked together by several inter- and intrachain disulfide bonds ( - Fig. 1A). Both the subunits and the chains are linked together by disulfide bonds and assembled to form at least 20 distinct independently folded domains. ${ }^{3}$ These domains are grouped into several structural regions, the central E region, two identical terminal $\mathrm{D}$ regions, and two $\alpha \mathrm{C}$ regions. Such nomenclature ${ }^{3}$ is based on the results of proteolytic cleavage of fibrinogen, during which the $\alpha \mathrm{C}$ regions are removed at the early stages of proteolysis, resulting in fragment $\mathrm{X}$, which is then split into two identical proteolytically resistant $\mathrm{D}$ fragments and one $\mathrm{E}$ fragment. These fragments derive from the $\mathrm{D}$ and $\mathrm{E}$ regions and correspond to the three nodules and connectors originally observed for the fibrinogen molecule by Hall and Slayter using electron microscopy (EM). ${ }^{4}$ Historically, these nodules were denoted as $\mathrm{D}$ and $\mathrm{E}$ domains, linearly arranged in a D-E-D manner. However, subsequent studies performed by differential scanning calorimetry (DSC) and EM revealed that the structure of the fibrinogen molecule is much more complex than the originally proposed trinodular model. ${ }^{4}$ Namely, EM studies visualized additional nodules in the fibrinogen molecule $e^{5-9}$ and DSC experiments identified several independently folded domains in its D and E regions ${ }^{10-12}$ (-Fig. 1B). The existence of multiple domains in fibrinogen has been confirmed later by X-ray analysis of the D and E fragments. ${ }^{13-15}$ Finally, X-ray studies established the crystal structure of native chicken fibrinogen, ${ }^{16,17}$ partially proteolyzed bovine fibrinogen, ${ }^{18}$ and human fibrinogen ${ }^{19}$ (-Fig. 1C). However, these studies did not resolve the structure of the $\alpha \mathrm{C}$ regions.

\section{Does the aC Region Form Ordered Structures?}

Before the crystal structure of the fibrinogen molecule was solved, the information about the structure of fibrinogen regions and domains came mainly from studies performed by sequence analysis, limited proteolysis, EM, and DSC. In our previous review, ${ }^{1}$ we summarized such information. Briefly, for a long time the $\mathrm{aC}$ regions were thought to be unordered forming so-called "free-swimming appendages." ${ }^{20}$ However, DSC experiments revealed the presence of compact ordered structures in the $\alpha \mathrm{C}$ regions ${ }^{10}$ (- Fig. 1B). Further, thermodynamic analysis of the unfolding process of these regions suggested that each of them forms independently folded domains and these domains interact with each other, forming a single structural block $^{11}$ (- Fig. 1D). Furthermore, sequence analysis revealed a high probability of secondary structure formation in the C-terminal halves of these regions ( - Fig. 2A), resulting in a suggestion that each of the two $\alpha \mathrm{C}-$ domains consists of a compact ordered structure attached to the bulk of the molecule with an extended flexible polypeptide junction ${ }^{11}$ (-Fig. 1D). In agreement, in a parallel EM study, Erickson and Fowler observed an extra nodule in the fibrinogen molecule that was absent in the early fibrin degradation product fragment $\mathrm{X}^{8}$ Since the $\mathrm{X}$ fragment differs from intact fibrinogen mainly by the absence of the C-terminal portions of the $\mathrm{A} \alpha$ chains ( $\alpha \mathrm{C}$ regions), they concluded that this extra nodule is formed by these portions and denoted it as $\alpha$ chain nodule ${ }^{8}$ (- Fig. 1E). Our subsequent EM study revealed that this extra nodule (we called it interacting $\alpha$ C-domains) is located near the central nodule $\mathrm{E}$ and is split into two entities ( $\alpha \mathrm{C}$-domains) in fibrin molecules. ${ }^{21}$ Based on these observations, ${ }^{21}$ as well as on our previous DSC experiments, ${ }^{11}$ we concluded that the $\alpha \mathrm{C}$ domains form compact ordered structures that interact intramolecularly with each other and with the central region of the molecule and dissociate from each other and from the central region upon conversion of fibrinogen into fibrin. ${ }^{1}$

Although DSC and EM studies described in detail in our previous review ${ }^{1}$ and briefly summarized above revealed that the $\alpha \mathrm{C}$ regions contain compact ordered structures, subsequent X-ray studies did not support this finding. 

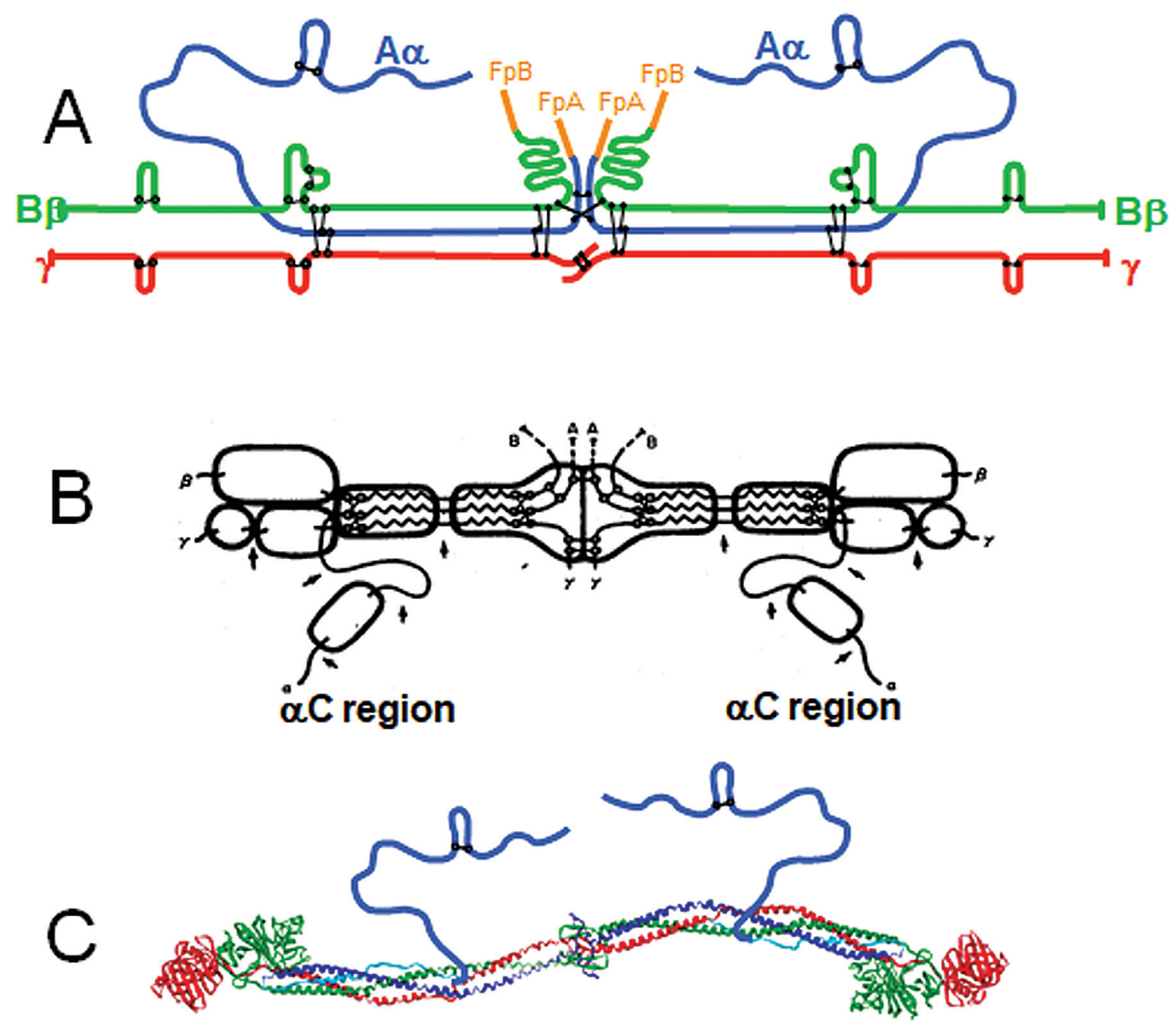

\section{$\alpha C$-domains}
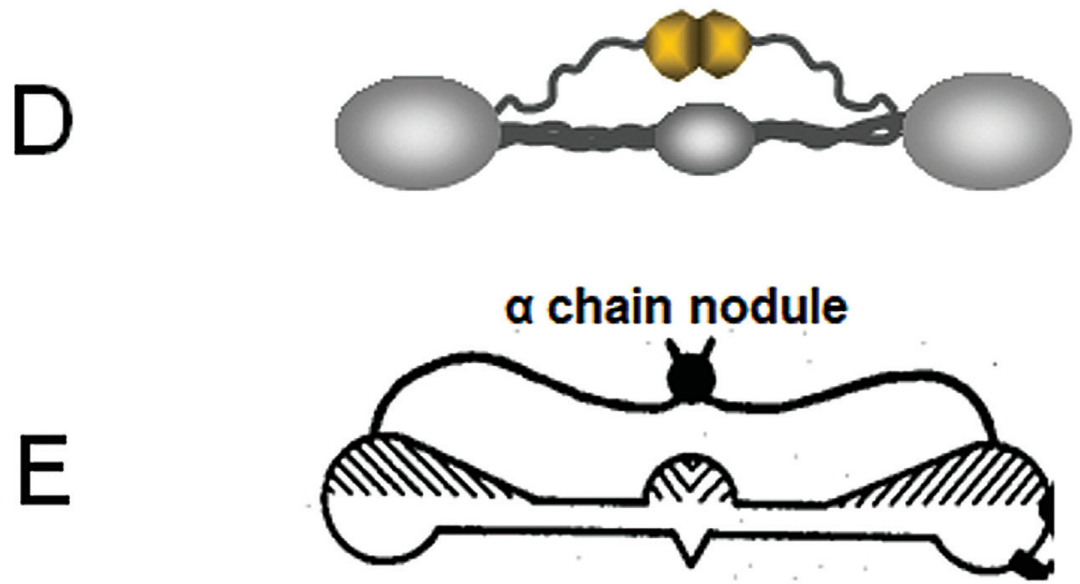

Fig. 1 (A) Polypeptide chain composition of the fibrinogen molecule. $A \alpha, B \beta$, and $y$ chains are in blue, green, and red colors, respectively, intraand inter-chain disulfide bonds are in black color; $\mathrm{FpA}$ and $\mathrm{FpB}$, fibrinopeptides $\mathrm{A}$ and $\mathrm{B}$, respectively, are in orange color. (B) Domain structure of the fibrinogen molecule derived from DSC study. ${ }^{10}$ (C) Ribbon diagram of the fibrinogen molecule based on its crystal structure. ${ }^{19}$ The $\alpha C$ regions, whose X-ray structure has not been defined, are shown in blue color. (D) Schematic representation of the interacting $\alpha \mathrm{C}$-domains in the fibrinogen molecule based on the results of DSC study. ${ }^{11}$ (E) Schematic representation of the $\alpha$ chain nodule in the fibrinogen molecule observed by electron microscopy. ${ }^{8}$ DSC, differential scanning calorimetry. 


\section{$\alpha \mathrm{C}$-connector}
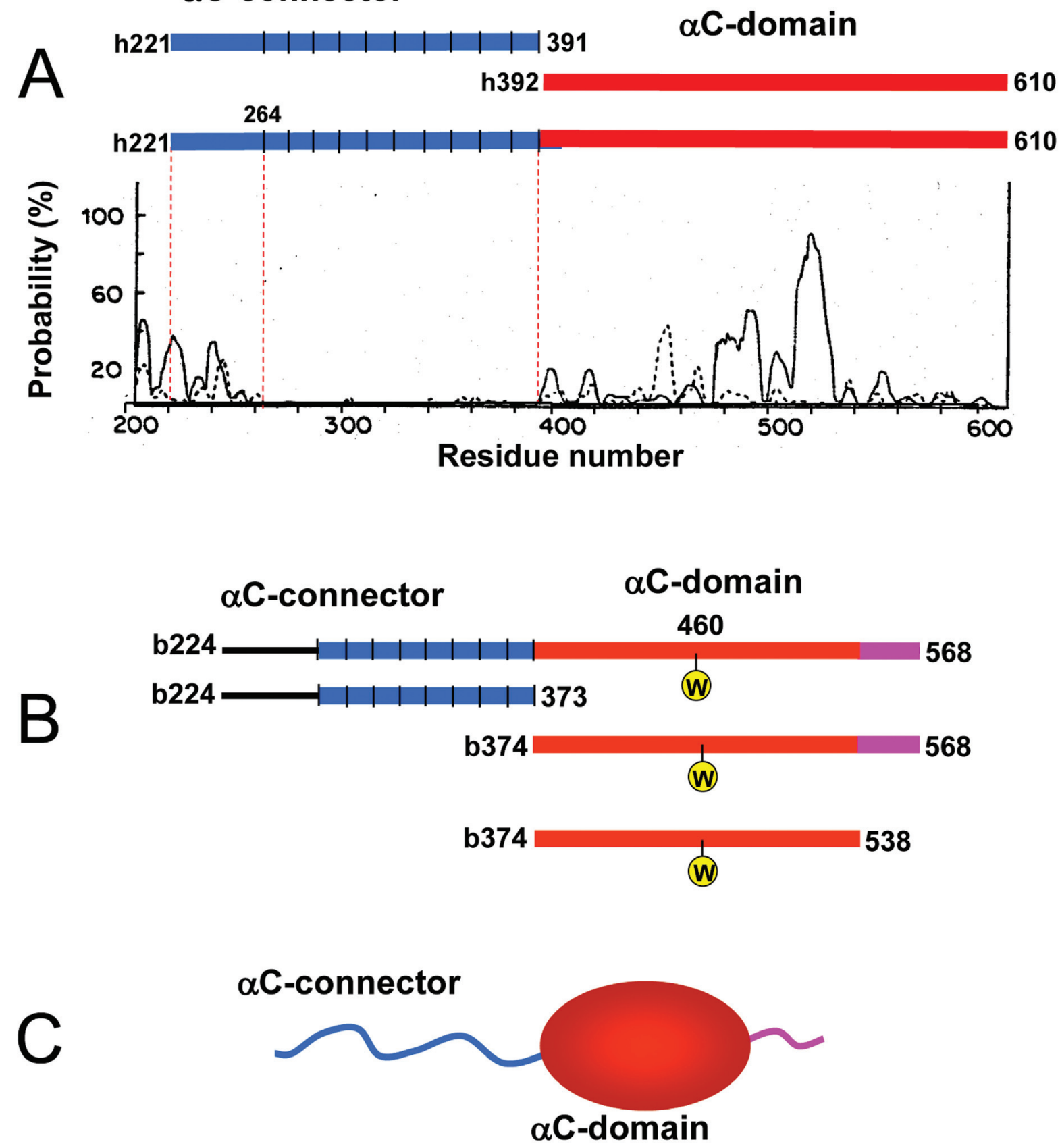

Fig. 2 (A) Probability of secondary structure formation for the C-terminal portion of the human Aa chain. ${ }^{11}$ Bars on the top represent the recombinant human fibrinogen $\alpha \mathrm{C}$ region (blue-red), $\alpha \mathrm{C}$-connector (blue), and $\alpha \mathrm{C}$-domain (red). (B) Schematic representation of the bovine fibrinogen $\alpha C$ region (blue-red), $\alpha C$-connector (blue), and $\alpha C$-domain (red). Vertical lines in the $\alpha \mathrm{C}$-connectors in both panels depict the boundaries of the 13-residue tandem repeats; the only tryptophan residue in the $\alpha \mathrm{C}$-domain is denoted by "W." (C) Schematic representation of the flexible $\alpha \mathrm{C}$-connector and compact $\alpha \mathrm{C}$-domain formed by the $\alpha \mathrm{C}$ region of the fibrinogen molecule.

Specifically, X-ray analysis of the crystal structure of native chicken fibrinogen $^{16,17}$ revealed that the $\alpha \mathrm{C}$ regions are not visible in electron density maps, leading to the conclusion that their structure is very disordered. ${ }^{17}$ This finding again raised a question about the folding status of the $\alpha \mathrm{C}$ regions, i.e., whether they indeed contain compact ordered structures, or they are unordered. The major problem with our results obtained by $\mathrm{EM}^{21}$ was that the EM technique allows us to observe the shape of a molecule but does not allow us to conclude whether a polypeptide in this shape is ordered or disordered. The major weakness of our results obtained by DSC was that we made a conclusion about compact ordered structures in the $\alpha \mathrm{C}$-domains based on indirect data. Namely, comparison of melting profiles of intact bovine fibrinogen and its fragment $X$ devoid of the $\alpha C$ regions revealed an extra low enthalpy heat absorption peak connected with unfolding 
of compact structures only in fibrinogen. Since no such peak was observed in the $\mathrm{X}$ fragment, we assigned this extra peak to melting (unfolding) of the $\alpha \mathrm{C}$-domains. ${ }^{10,11}$ At the same time, such a heat absorption peak was not observed in intact human fibrinogen. ${ }^{22}$ Thus, measurements with isolated $\alpha \mathrm{C}$ regions were required to obtain direct evidence for the presence of compact structures in these regions.

\section{Experiments with Isolated $\alpha$ C-Domains Confirmed the Presence of Ordered Structures}

The two fibrinogen $\alpha \mathrm{C}$ regions, each made up of the $\mathrm{C}$ terminal portion of the A $\alpha$ chain, have different size in fibrinogens from different species. For example, in human fibrinogen they encompass amino acid residues A $221-610$, and in bovine fibrinogen they are shorter, including residues A 2224-568 (bovine numbering). ${ }^{1,23}$ These regions are easily removed from fibrinogen during initial stages of plasmin cleavage and, in contrast to the $\mathrm{D}$ and $\mathrm{E}$ fragments, which are resistant to further proteolysis, they are cleaved into smaller fragments. Such high susceptibility to proteolysis complicated their preparation in quantities sufficient for their structural/functional study. In addition, the intrinsic C-terminal heterogeneity of the A $\alpha$ chains ${ }^{24}$ and the presence of multiple plasmin cleavage sites in the C-terminal portions of the $\mathrm{A} \alpha$ chains $^{25}$ prevented preparation of homogenous $\alpha \mathrm{C}$ fragments. For example, the $40-\mathrm{kDa} \alpha \mathrm{C}$-fragments prepared from early plasmin digests of bovine and human fibrinogens were heterogeneous and their final yields were very low. ${ }^{26,27}$

To overcome these problems, we first expressed and characterized the recombinant $45-\mathrm{kDa} \alpha \mathrm{C}$-fragment corresponding to the $\alpha \mathrm{C}$ region of human fibrinogen. ${ }^{28}$ While covalent cross-linking of this fragment by factor XIIIa was obvious, no regular structures were observed by circular dichroism (CD), most probably because the fragment was expressed in Escherichia coli and was purified from the inclusion bodies that were dissolved in denaturing concentrations of urea. ${ }^{28}$ To find appropriate conditions for refolding, we next expressed in E. coli recombinant fragments corresponding to the bovine and human $\alpha \mathrm{C}$ regions and their $\mathrm{N}$-terminal and C-terminal halves ${ }^{23}$ ( - Fig. 2A, B). The major reason for the expression of bovine $\alpha \mathrm{C}$-fragments was that the bovine $\alpha \mathrm{C}$ region, in contrast to the human one, contains a single tryptophan residue (Trp460) in its C-terminal half (-Fig. 2B). This allowed the refolding to be monitored by fluorescence spectroscopy which requires only very small amounts of protein per experiment. This method enabled us to test various refolding conditions for the bovine $\alpha \mathrm{C}$-fragments and to develop a protocol for their refolding. ${ }^{23}$ This protocol was then used for refolding of human $\alpha \mathrm{C}$ fragments. ${ }^{23}$

Our experiments with the refolded recombinant bovine $\alpha \mathrm{C}$ fragments performed by fluorescence spectroscopy revealed prominent sigmoidal transitions upon heating of the full-length $\alpha \mathrm{C}$ region fragment and its C-terminal half, which reflected cooperative unfolding of compact structures, while the $\mathrm{N}$-terminal half exhibited no such transition. ${ }^{23}$
These findings were confirmed by CD and by DSC. ${ }^{23}$ Similar results in CD and DSC experiments were obtained with the recombinant human $\alpha \mathrm{C}$-fragments. ${ }^{23}$ Altogether, these results directly confirmed our original conclusion ${ }^{10,11}$ that the $\alpha \mathrm{C}$-domains contain compact ordered structures. Consistent with these results, subsequent atomic force microscopy studies of fibrin(ogen), using a modified graphite surface and unique super-sharp cantilevers, clearly showed the $\alpha \mathrm{C}$ regions, with compact $\alpha \mathrm{C}$-domains. ${ }^{29}$ Moreover, measurements of the $\alpha \mathrm{C}$-connector length were considerably less than what would be expected of an unfolded polypeptide chain, ${ }^{29}$ suggesting some folded structure.

\section{aC Region or aC-Domain?}

The C-terminal portions of the fibrinogen A $\alpha$ chains that are easily removed by proteolysis were originally called the $\alpha \mathrm{C}-$ domains. ${ }^{111,30}$ However, subsequent study $^{23}$ described above revealed that this terminology does not reflect the structural organization of these regions, since each of them consists of two structurally very distinct portions. Namely, the C-terminal half of this region forms an independently folded compact cooperative unit (domain) while the $\mathrm{N}$ terminal half forms a flexible region tethering the compact globular part to the bulk of the molecule ( $\mathbf{- F i g} . \mathbf{2 C}$ ). Therefore, it was proposed to refer to the compact part as the $\alpha \mathrm{C}$ domain and to the flexible part as the $\alpha \mathrm{C}$-connector. ${ }^{23}$ Such terminology has been approved by the Subcommittee on Fibrinogen and Factor XIII of the International Society on Thrombosis and Haemostasis Scientific and Standardization Committee ${ }^{3}$ and will be further used in this review. In addition, the recombinant fragments containing human and bovine $\alpha \mathrm{C}$ regions and their $\mathrm{N}$ - and C-terminal halves (-Fig. 2) will be called for simplicity as follows: human $\alpha \mathrm{C}$

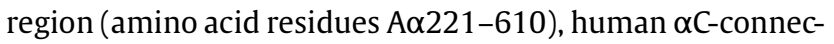

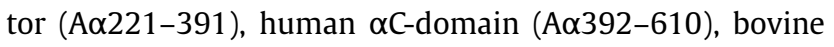

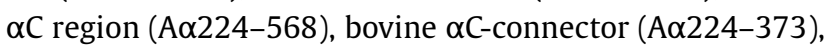
bovine $\alpha \mathrm{C}$-domain (A $\alpha 374-568)$, and bovine truncated $\alpha \mathrm{C}$ -

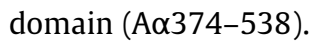

\section{Structure of the aC-Connector}

Different sizes of the fibrinogen $\alpha \mathrm{C}$ regions in different animal species are mainly a result of the lengths of their $\alpha \mathrm{C}$-connectors. The connector regions of bovine and human fibrinogens (residues 221-391 and 224-373, respectively) start with a 43-45 residue segment followed by several 13residue internal tandem repeats, 10 in human and 8 in bovine $^{23}$ (-Fig. 2). There are 13 such repeats in monkey

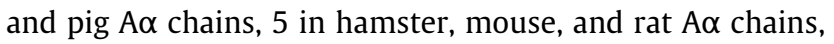
and no such repeats in chicken $A \alpha$ chains $^{31}$. Most of these repeats are rich in Gly and Pro. Because it was shown that such tandemly repeated Pro-rich sequences found in many proteins may adopt the extended left-handed helical conformation known as poly(L-proline) type II (PPII), ${ }^{32}$ we hypothesized in our previous review ${ }^{1}$ that the $\alpha \mathrm{C}$-connectors may contain such a conformation. In agreement with this hypothesis, our subsequent study performed by CD and DSC 
revealed that the recombinant bovine and human $\alpha \mathrm{C}$-connectors, as well as a synthetic peptide mimicking the sixth tandem repeat of the human connector, exhibit some features characteristic for PPII. ${ }^{23}$ Namely, all three connector species exhibited CD spectra, temperature-induced behavior of CD signals at $222 \mathrm{~nm}$, and heat capacity function changes ${ }^{23}$ similar to those reported for the PPII conformation. ${ }^{33,34}$ Since such a conformation was suggested to be involved in protein-protein interaction, ${ }^{32}$ one can speculate that tandem repeats of the $\alpha \mathrm{C}$-connectors are not "free-swimming," as presented in - Fig. 1(B-E), but may interact with the coiledcoil and/or other structures of the fibrinogen molecule. In agreement, the fact that a fibrin-specific monoclonal antibody obtained to fibrin fragment B $\beta 118-134$ does not interact with fibrinogen ${ }^{35}$ suggests that this section of the $B \beta$ chain, which is located in the coiled-coil domain of the central region $\mathrm{E}^{3}$ may interact with and be covered by the $\alpha C$-connector. This speculation needs to be further tested.

It should be noted that crystal structures of human fibrinogen and its $D$ fragment ${ }^{13,19}$ revealed that the $A \alpha$ chain in each fibrinogen subunit makes a turn after Cys165 of the second disulfide ring, which restricts the triple-helical coiled-coil region, to form a fourth $\alpha$-helical strand of the coiled-coil. This $\alpha$-helix is formed by the A $\alpha$ chain residues 176-188 and nestles in the groove of the triple helix between the $A \alpha$ and $B \beta$ chains. ${ }^{13}$ It directs the $C$-terminal portion of the A $\alpha$ chain toward the central region of the molecule and represents most probably a part of the coiled-coil domain of the $\mathrm{D}$ region. ${ }^{3}$ The structure of the remaining portion of this chain, which contains at least four cleavage sites for plasmin at $\operatorname{Arg} 198, \operatorname{Arg} 200$, Lys207, and Lys220, ${ }^{25}$ and that of the 4345 residue segment of the $\alpha \mathrm{C}$-connector preceding its tandem repeats, have not been defined. However, the experiments with specific anti-A $\alpha$ chain monoclonal antibodies, which revealed that an epitope within the A $2220-230$ region becomes newly exposed in the isolated $\alpha \mathrm{C}$ fragment as a result of plasmin cleavage at residues $A \alpha 219-220,{ }^{27}$ suggests that this region may form an ordered structure that may interact with (and "stick" to) the coiled-coil domains. This speculation is in good agreement with the secondary structure prediction results ${ }^{11}$ that indicate a high probability of regular structure formation in the 200-240 region of the A $\alpha$ chain (-Fig. 2A).

\section{NMR Study of $\alpha$ C-Domain Fragments}

Although the results described above clearly indicated the presence of compact regular structures in the bovine and human $\alpha C$-domains, their three-dimensional (3D) structure remained unknown. X-ray analysis can provide direct information about 3D structure of proteins; however, our efforts to crystallize the recombinant $\alpha C$-fragments, as well as those performed by others, ${ }^{36,37}$ had been unsuccessful. Therefore, to establish the 3D structure of the $\alpha \mathrm{C}$-domain, we turned to nuclear magnetic resonance (NMR), which allows determination of high-resolution protein structures in solution. We first focused on NMR study of the bovine $\alpha \mathrm{C}$-fragment including amino acid residues A $\alpha 374-538$, which represent a truncated $\alpha$ C-domain (-Figs. 2B and 3A). This fragment was selected because it exhibited the most pronounced heatinduced unfolding transition in our fluorescence study, ${ }^{23}$ and computer analysis of the probability of secondary structure

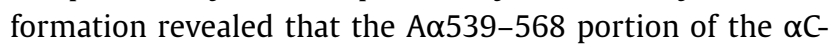
domain, which is missing in this fragment, has a low probability of forming regular structures ${ }^{11}$ ( - Fig. 2A). Our study of this fragment performed in collaboration with Dr. Nico Tjandra (National Institutes of Health/National Heart, Lung, and Blood Institute) revealed two antiparallel $\beta$-strands forming a $\beta$-hairpin restricted at the end by a Cys423-Cys453 disulfide linkage. ${ }^{38}$ In addition, an ordered collapsed hydrophobic region next to the hairpin, which includes approximately 30 amino acid residues, has also been identified. ${ }^{38}$ However, the 3D structure of this collapsed region was not determined due to the resonance ambiguity arising from the disordered portions of this fragment. To overcome this problem, we expressed a shorter fragment, bA $406-483$ ( - Fig. 3A), devoid of such disordered portions, and established its complete NMR solution structure. ${ }^{39}$ The structure revealed a second loose $\beta$-hairpin in the collapsed hydrophobic region, yielding a parallel/antiparallel $2{ }_{\uparrow} 1_{\downarrow} 3 \downarrow_{\downarrow} 4_{\uparrow} \beta$ sheet (-Fig. 3B).
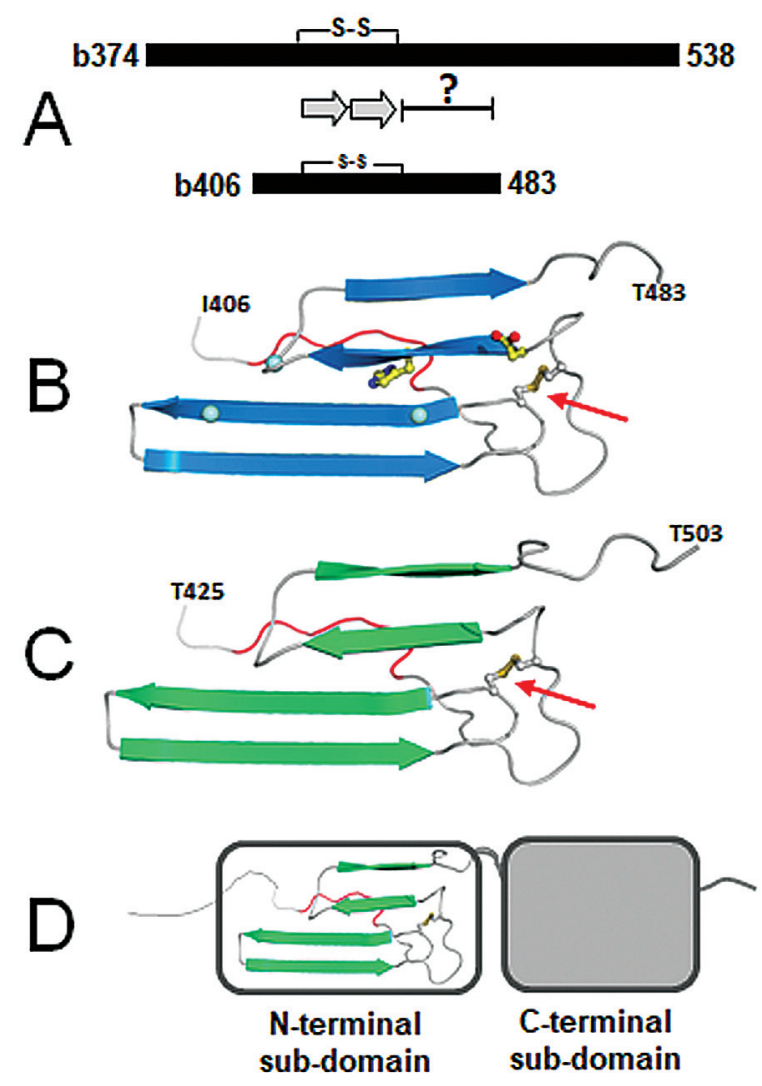

Fig. 3 (A) Schematic representation of the bovine fibrinogen $\alpha C$ fragments b374-538 (top bar) and b406-483 (bottom bar). Arrows indicate two $\beta$-strands identified by NMR; the collapsed hydrophobic region is denoted by "?." (B) NMR solution structure of the bovine b406-483 $\alpha$ C-fragment. ${ }^{39}$ (C) Homology model of the human h425$503 \alpha$ C-fragment. ${ }^{40}$ (D) Subdomain structure of the $\alpha C$-domain. ${ }^{40}$ Arrows in panels (B) and (C) show location of a Cys423-Cys-453 disulfide linkage. NMR, nuclear magnetic resonance. 
The same approach had been used to investigate the NMR solution structure of the human $\alpha C$-domain. Namely, we tested the hA $\alpha 425-503$ fragment, which is homologous to the bovine bA $406-483$ fragment, by NMR and found that the peak dispersion of its ${ }^{15} \mathrm{~N}$-HSQC spectrum is similar to that of its bovine counterpart, suggesting that their overall folding is similar. ${ }^{40}$ However, the intensities of the peaks were lower than those of bA $4406-483$, and NMR relaxation data revealed that residues corresponding to the second $\beta$ hairpin are more mobile in the human fragment and thereby form a less stable conformation than those in the bovine counterpart. These results complicated the analysis of the NMR spectral data and precluded establishment of the complete structure of the human $h A \alpha 425-503$ fragment. Therefore, to clarify its structural organization, we performed homology modeling of the 3D structure of human $\mathrm{hA} \alpha 425-503$, using the structure of bovine bA $\alpha 406-483$ as a template. ${ }^{40}$ The resultant model of the hA $\alpha 425-503$ fragment presented in - Fig. $3 \mathbf{C}$ confirmed the structural similarity between the bovine and human fragments. ${ }^{40}$

It should be noted that analysis of amino acid sequences in the $\alpha \mathrm{C}$-domains of 11 species, from human to such distant species as zebrafish, frog (Xenopus), and chicken, revealed that they all preserve a disulfide-linked loop forming the first $\beta$-hairpin in the bovine $\alpha \mathrm{C}$-domain. ${ }^{38}$ The fact that in all these species this loop has a very similar size (29-32 residues) and relatively high conservation ( $\sim 60 \%$ identical residues) strongly suggests that it should form a $\beta$-hairpin similar to that of the bovine $\alpha \mathrm{C}$-domain. ${ }^{38,39}$ As to the region that forms a second $\beta$-hairpin in the bovine $\alpha C$-domain, it has a higher

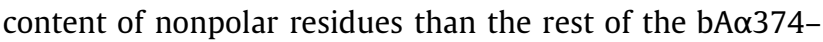
538 fragment ( 40 vs. $31 \%$ ). The content of nonpolar residues in this region in other species is even higher (55\% in human, $56 \%$ in monkey, and $59 \%$ in hamster). ${ }^{38}$ Thus, one can expect that this region forms a second $\beta$-hairpin in the $\alpha \mathrm{C}$-domains of other species mentioned above, although its stability may be different from that of the bovine $\alpha \mathrm{C}$-domain.

\section{Identification of Additional Compact Structures in the $\alpha C$-Domains}

The bovine bo406-483 fragment, whose NMR structure has been determined, ${ }^{39}$ and its human counterpart, the hA $\alpha 425-503$ fragment, whose 3D structure was modeled, ${ }^{40}$ both form a compact, independently folded domain. However, these fragments represent only approximately $40 \%$ of the corresponding full-length $\alpha \mathrm{C}$-domains (-Fig. 3A). The structure of the remaining portions of these domains had been clarified after our finding that the recombinant bovine and human $\alpha \mathrm{C}$-domain fragments form soluble oligomers. $^{39,40}$ Namely, it was found that while monomeric fractions of these fragments exhibit $C D$ spectra indicating a considerable amount of unordered structures, analysis of CD spectra of their oligomeric fractions revealed a significant increase in their regular structures, mostly an increase in $\beta$-sheet content. ${ }^{40,41}$ Such an increase can only be explained by formation of additional regular structures in the $\alpha \mathrm{C}$-domain upon its oligomerization. Thus, in addition to the compact domain formed by the N-terminal half of the $\alpha$ C-domain (-Fig. 3B, C), its C-terminal half also forms a compact regular structure that can be considered as an additional domain (-Fig. 3D). To preserve the recommended nomenclature for fibrinogen structure, ${ }^{3}$ we proposed to denote them as $\mathrm{N}$ - and C-terminal subdomains of the $\alpha \mathrm{C}$-domain. ${ }^{40}$ It should be noted that the C-terminal subdomain forms stable compact structures only in $\alpha C$ domain oligomers and is unstable and unfolded in the isolated $\alpha C$-domain fragments.

\section{Intra- and Intermolecular Interactions of the aC-Domains in Fibrinogen and Fibrin}

Our early studies, which revealed that the $\alpha \mathrm{C}$-domains interact with each other in fibrinogen ${ }^{11}$ and participate in fibrin polymerization, ${ }^{30}$ resulted in the hypothesis that these domains switch from intramolecular to intermolecular interactions upon conversion of fibrinogen to fibrin to promote an ordered fibrin assembly. ${ }^{30}$ Subsequent EM study and turbidity measurements, ${ }^{21,26}$ which are summarized in our previous review, ${ }^{1}$ provided some experimental evidence supporting this hypothesis. Briefly, EM experiments with an $\alpha \mathrm{C}$-domain-specific monoclonal antibody revealed that the extra nodule representing two interacting $\alpha C$-domains is mainly located near the central nodule of the fibrinogen molecule, suggesting that these domains interact with the central fibrinogen E region. ${ }^{21}$ Further, we observed dissociation of the $\alpha \mathrm{C}$-domains from each other and from the $\mathrm{E}$ region upon removal of $\mathrm{FpA}$ and $\mathrm{FpB}$, suggesting that these fibrinopeptides play an important role in the interaction of the $\alpha C$-domains with the central part of the molecule. $^{21}$ We also observed intermolecular interaction between the $\alpha \mathrm{C}$-domains and found that a proteolytically prepared $40-\mathrm{kDa} \alpha \mathrm{C}$-domain fragment can bind to the $\alpha \mathrm{C}$ domains of fibrin and decrease the rate of fibrin assembly. $^{21,26}$

The results described above allowed us to further develop our original intra- to intermolecular switch hypothesis. Namely, we suggested that in fibrinogen two $\alpha \mathrm{C}$-domains interact intramolecularly with each other and with the central region $\mathrm{E}$ of the molecule, and the removal of fibrinopeptides by thrombin upon fibrinogen to fibrin conversion results in the dissociation of the $\alpha \mathrm{C}$-domains from the central region $\mathrm{E}$ and from each other and their switch to intermolecular $\alpha C-\alpha C$ interactions that promote fibrin assembly. ${ }^{1}$ This hypothesis required further experimental conformation since most of the evidence for the interactions of the $\alpha \mathrm{C}$-domains came from $\mathrm{EM}$ studies of fibrinogen and fibrin. Thus, more direct experiments with the isolated $\alpha \mathrm{C}$-domain fragments demonstrating these interactions in solution were required.

\section{Direct Evidence for Specific Interactions of the Fibrinogen $\alpha C$-Domains with Each Other and with the Central E Region}

The first hint for the intermolecular interactions between the isolated $\alpha \mathrm{C}$-domains was obtained with the proteolytically 
prepared bovine $40-\mathrm{kDa} \alpha \mathrm{C}$-fragment corresponding to the truncated bovine $\alpha \mathrm{C}$ region. This fragment formed long, almost linear, branching arrays as revealed by EM. ${ }^{21}$ Formation of similar ordered arrays was also observed by EM with its recombinant analog, the bovine bA $\alpha 224-538$ fragment, as well as with the recombinant full-length version of the bovine $\alpha C$ region, the bA $\alpha 224-568$ fragment. ${ }^{42}$ These fragments were monomeric at acidic $\mathrm{pH}$ and formed ordered oligomers when they were transferred from acidic to neutral $\mathrm{pH}^{42}$ However, when these fragments kept at neutral $\mathrm{pH}$ were diluted with the neutral EM buffer, only monomeric species were observed. ${ }^{42}$ Moreover, experiments performed by the multi-angle light scattering technique and sedimentation velocity revealed that both fragments are monomeric at neutral $\mathrm{pH} .{ }^{42}$ This apparent paradox was solved after we found that various $\alpha C$-domain fragments form soluble oligomers. First, using size-exclusion chromatography and analytical ultracentrifugation, we observed formation of soluble oligomers by the

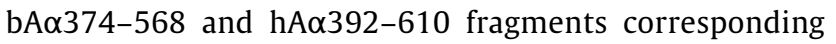
to the bovine and human $\alpha \mathrm{C}$-domains, respectively. ${ }^{40}$ Oligomerization of these fragments was concentrationdependent and reversible, suggesting highly specific interactions between the $\alpha \mathrm{C}$-domains upon oligomer formation. Second, our experiments revealed that the recombinant A $\alpha 221-610$ fragment corresponding to the human $\alpha C$ region also forms ordered oligomers in a concentration-dependent and reversible manner. ${ }^{41}$ Finally, our experiments using optical trap-based force spectroscopy further confirmed and quantified intermolecular interaction between the isolated human $\alpha \mathrm{C}$-domains. ${ }^{43}$ The experiments also revealed interactions between the isolated human $\alpha C$-domains and the isolated central $\mathrm{E}$ region of fibrinogen (NDSK fragment), which mainly occurs through $\mathrm{FpB}$, although FpA are also involved in this interaction. ${ }^{43}$ Thus, all experiments described above provided direct evidence for the interactions between the $\alpha \mathrm{C}$-domains and between the $\alpha \mathrm{C}$-domains and the central region of the fibrinogen molecule. However, the molecular mechanism underlying the intra- to intermolecular switch of these interactions remained unclear.

\section{Unraveling the Molecular Mechanism of $\alpha C$ Polymer Formation in Fibrin}

Conversion of monomeric fibrinogen into polymeric fibrin occurs through intermolecular noncovalent interactions between complementary polymerization sites called knobs and holes ${ }^{3}$ (knob-hole interactions) and is accompanied by covalent cross-linking with a specific plasma transglutaminase, factor XIIIa. Besides intermolecular cross-linking of fibrin $\gamma$ chains $(\gamma-\gamma$ cross-linking resulting in $\gamma$ chain dimers), factor XIIIa also cross-links fibrin's $\alpha$ chains. Analysis of cross-linked fibrin revealed that cross-linking of the $\alpha$ chains in polymeric fibrin results in formation of $\alpha$ chain polymers. ${ }^{44-46}$ This observation suggested that the $\alpha C$ domains are closely spaced in polymeric fibrin and may interact with each other to form such polymers, even without factor XIIIa cross-linking. Indeed, our subsequent studies described above revealed that the isolated $\alpha \mathrm{C}$ domains, as well as the isolated $\alpha C$ regions, interact with each other to form soluble ordered oligomers. ${ }^{40,41}$ Detailed analysis of EM images of XIIIa-cross-linked oligomers formed by the isolated $\alpha C$ regions (the hA $\alpha 221-610$ fragment) revealed long thin filaments with the width of about twice the diameter of individual $\alpha C$ monomers $^{41}$ (-Fig. 4). Such oligomers had an apparently ordered structure and mimicked the structure and interactions of the $\alpha \mathrm{C}$-domains in fibrin. ${ }^{40,41}$

To clarify the molecular mechanism underlying formation of $\alpha C$ polymers in fibrin, we tested interactions between the recombinant $\alpha \mathrm{C}$-domain and the $\alpha \mathrm{C}$-connector fragments, and interaction of the recombinant $\mathrm{N}$ - and $\mathrm{C}$ terminal subdomain fragments with each other and with the $\alpha \mathrm{C}$-connector fragment. The experiments performed by optical trap-based force spectroscopy revealed that the $\alpha \mathrm{C}$ domain interacts with the $\alpha \mathrm{C}$-connector. ${ }^{47}$ Further, using the same technique, it was found that the N-terminal subdomains interact with each other, while no interaction was found between the C-terminal subdomains, ${ }^{47}$ in agreement with the previous observations. ${ }^{39,40}$ Finally, these experiments revealed that the C-terminal subdomain fragment interacts with the $\alpha \mathrm{C}$-connector while the $\mathrm{N}$-terminal subdomain fragment does not. ${ }^{47}$ Altogether, these findings indicate that formation of ordered oligomers by the $\alpha \mathrm{C}$ regions in fibrin polymer occurs mainly through the homophilic interaction between their N-terminal subdomains. In addition, interactions between the C-terminal subdomains and the $\alpha \mathrm{C}$-connectors contribute to self-association of the $\alpha C$ regions in fibrin.
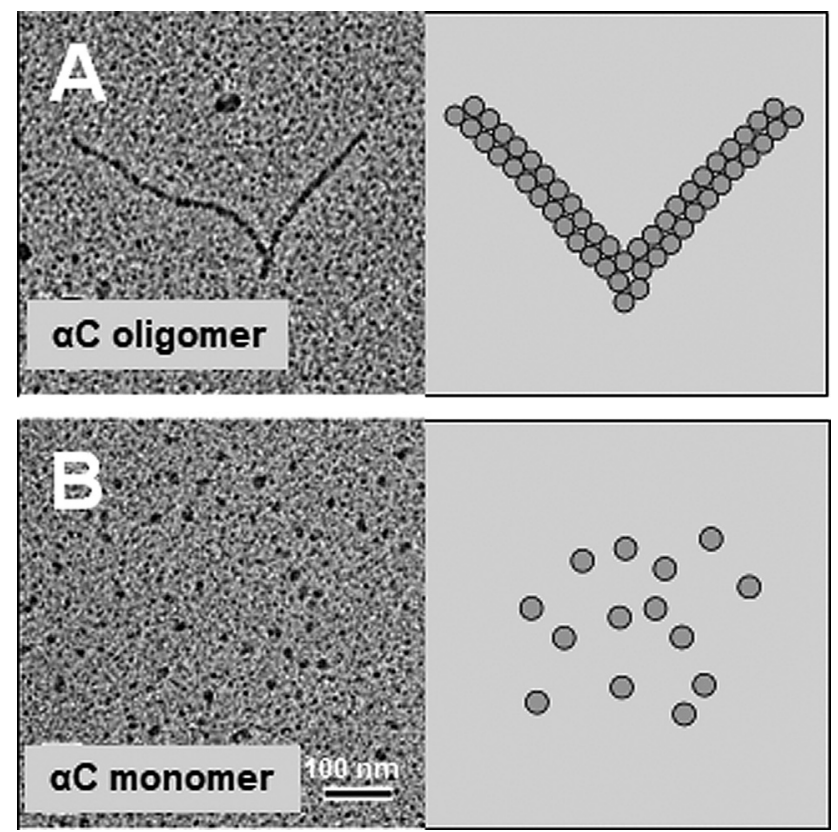

Fig. 4 Electron micrographs of rotary-shadowed samples of cross-

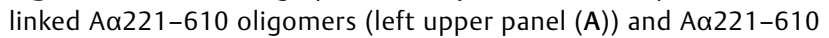
monomers (left lower panel (B)). ${ }^{41}$ Schematic diagrams of the observed structures are shown on the right panels. 


\section{Modeling Self-Association of the $\mathbf{N}$-terminal Subdomains}

Although oligomers formed by the $\alpha C$ fragments ${ }^{40,41}$ mimic the structure of the $\alpha \mathrm{C}$-domains in fibrin, their 3D structure cannot be established by NMR due to their large size. They also cannot be crystallized for X-ray analysis due to their heterogeneity. Therefore, to further clarify the molecular mechanism of $\alpha$ polymer formation, we performed molecular modeling that was based on the two major findings. First, we found that formation of oligomers by the $\alpha \mathrm{C}$-domains occurs mainly through homophilic interactions between their $\mathrm{N}$-terminal subdomains whose 3D structure has been established. ${ }^{39,40}$ Second, we found that the stability of monomeric forms of the bovine A $\alpha 406-483$ and human A $\alpha 25-503$ fragments corresponding to these N-terminal subdomains is very low, while that of their oligomeric forms is significantly increased. ${ }^{39,40}$ In fact, our unfolding experiments revealed that these fragments are intrinsically unstable and at physiological temperatures should be unfolded. ${ }^{39,40}$ Therefore, all NMR measurements were performed at $9^{\circ} \mathrm{C}$ to preserve their structure in our NMR studies. ${ }^{38-40}$

The very low stability of the monomeric forms of the bovine A $\alpha 406-483$ and human A $\alpha 425-503$ fragments is connected with their structure that includes a mixed fourstranded parallel/antiparallel $2_{\uparrow} 1_{\downarrow} 3_{\downarrow} 4_{\uparrow} \beta$-sheet in which two $\beta$-hairpins, $2_{\uparrow} 1_{\downarrow}$ and $3 \downarrow 4_{\uparrow}$, interact through parallel $\beta$ strands $1 \downarrow 3 \downarrow$ ( topology is unstable because the involvement of two parallel $\beta$-strands in the middle in both parallel and antiparallel hydrogen bonding may impose serious stress on the geometry of such a $\beta$-sheet, rendering the whole structure less stable. ${ }^{48}$ Thus, to explain a significant stabilization of these fragments upon their oligomerization, we hypothesized that their $\beta$-hairpins switch their orientation to become a part of an antiparallel $\beta$-sheet whose stability should be much higher than that of the four-stranded parallel/ antiparallel $\beta$-sheet. ${ }^{39}$ This can be achieved by a 3D domain swapping, which was proposed as a mechanism for formation of dimers or oligomers, ${ }^{49-52}$ namely, by swapping $\beta$ hairpins of the monomeric $\alpha C$ fragments to arrange all their $\beta$-strands in an antiparallel fashion. ${ }^{39}$ Molecular modeling aimed at evaluating the feasibility of the hypothesized $\beta$-hairpin swapping revealed that from a steric point of view such swapping is possible. ${ }^{47}$ Furthermore, among several alternative models analyzed, the one presented in - Fig. $\mathbf{5 A}$ is the most probable due to the lowest energy toll for such swapping. ${ }^{47}$ In this model, the swapping upon dimerization/oligomerization occurs by displacing two $\beta$ hairpins aside in the parallel/antiparallel $\beta$-sheet forming each fragment and replacing them with their counterparts from the interacting partner to form antiparallel $\beta$-sheets. The resulting dimer or oligomer, in which the $\beta$-hairpins of the neighboring $\mathrm{N}$-terminal subdomains are interlocked in a zipper-type manner, contains $\beta$-sheets packed closely "face-to-face" resembling the topology of a $\beta$-sandwich structural motif found in many proteins. Such a structure is stabilized by linear hydrogen bonds typical for an anti-

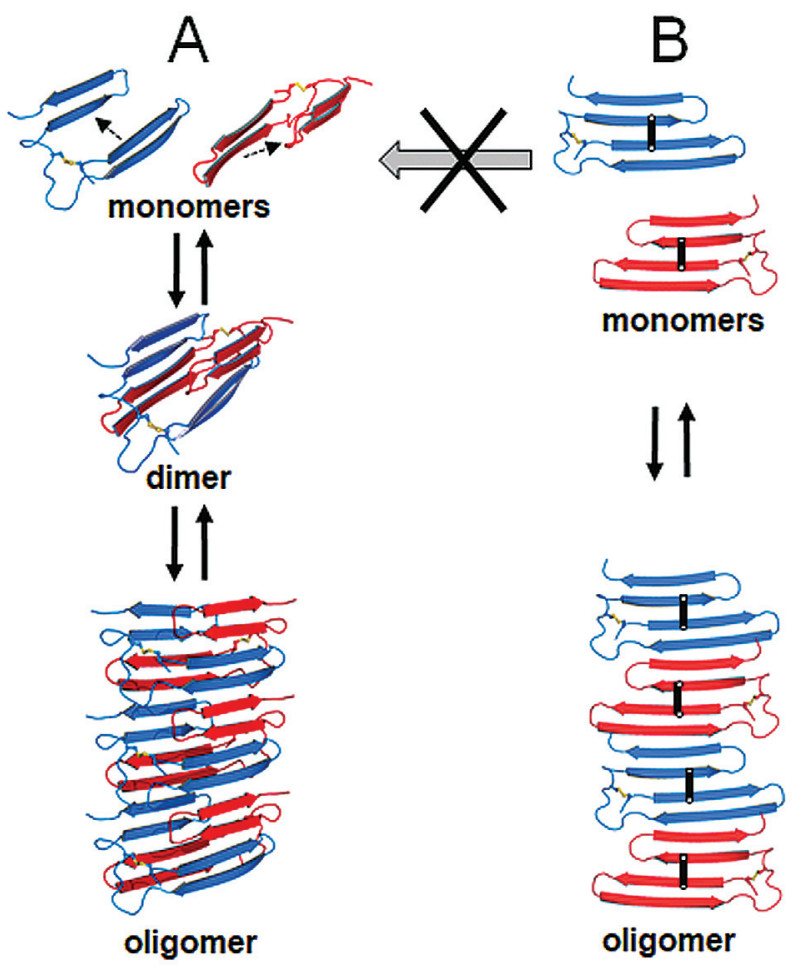

Fig. 5 Ribbon diagrams representing the proposed mechanism of dimer or oligomer formation by the N-terminal subdomains of the $\alpha C$ domains based on the results of molecular modeling. ${ }^{47}$ (A) Two monomeric N-terminal subdomains shown in red and blue for clarity, in which two $\beta$-hairpins are displaced aside (top), form a dimer (middle) and an oligomer (bottom). The displacement of the $\beta$-hairpins is shown by short, dashed arrows. (B) Two monomeric N-terminal subdomains "locked" in the $2 \uparrow 1_{\downarrow} 3_{\downarrow} 4_{\uparrow} \beta$-sheet conformation by the disulfide bonds shown by black vertical bars (top) can form only sideto-side oligomers (bottom).

parallel $\beta$-sheet and by hydrophobic interactions in the sandwich core. ${ }^{47}$

To experimentally test our $\beta$-hairpin swapping hypothesis, we first "locked" the structure of the A $\alpha 406-483$ fragment representing the $\mathrm{N}$-terminal subdomain in the $2{ }_{\uparrow} 1_{\downarrow} 3 \downarrow 4_{\uparrow} \beta$ sheet conformation to prevent the hypothesized swapping of the $\beta$-hairpins. This was done by introducing a disulfide bond between $\beta$-strands $1_{\downarrow}$ and $3_{\downarrow}$ (-Fig. 5B) using site-directed mutagenesis. ${ }^{47}$ Next, we compared the ability of the wild-type A 4406-483 and its "disulfide-locked" mutant to form oligomers and to be stabilized upon oligomerization. The experiments revealed that the wild-type A $\alpha 06-483$ formed oligomers in a concentration-dependent manner and its thermal stability was significantly increased upon oligomer formation, as expected. In contrast, formation of oligomers by the "disulfide-locked" mutant was much slower and to a lesser extent. Moreover, its oligomerization was not accompanied by thermal stabilization of its structure, ${ }^{47}$ most probably because its oligomers were formed by end-to-end interactions, as shown in - Fig. 5B, and a structurally unfavorable parallel/antiparallel conformation of the $\beta$-strands in such oligomers was still preserved. These results provided experimental support for the above-mentioned $\beta$-hairpin swapping hypothesis. 


\section{Current View on the Structure of the $\alpha C$ - Domains in Fibrinogen and the Molecular Mechanism of Their Intra- to Intermolecular Switch upon Conversion of Fibrinogen to Fibrin}

The information obtained since our previous review ${ }^{1}$ and described above allows us to better understand the structure and interactions of the $\alpha \mathrm{C}$-domains in the fibrinogen molecule and to clarify the molecular mechanism of the intra-tointermolecular switch of the $\alpha \mathrm{C}$-domains upon conversion of fibrinogen to fibrin. According to our current view, each fibrinogen $\alpha \mathrm{C}$-domain consists of the $\mathrm{N}$ - and C-terminal subdomains that are folded independently and contain mainly $\beta$-sheet structures ${ }^{40}$ ( - Fig. 3D). While the isolated $\alpha C$-domains and their subdomains are intrinsically unstable, they are stabilized in the fibrinogen molecule by the interaction with each other and with the central part of the molecule (-Fig. 6A). The interaction of the $\alpha \mathrm{C}$-domains with each other to form a dimer occurs mainly through their $\mathrm{N}$-terminal subdomains and involves, most probably, the $\beta$ hairpin swapping mechanism (-Fig. 5A). Their interaction with the central part of the molecule involves FpA and $\mathrm{FpB}^{21,43}$ In addition, their C-terminal subdomains may interact with the $\alpha C$-connectors. ${ }^{47}$

Based on the $K_{\mathrm{d}}$ values of 12 and $38.3 \mu \mathrm{M}$ determined for the interaction of isolated human and bovine $\alpha \mathrm{C}$-domain fragments, respectively, ${ }^{40}$ the affinity of the $\alpha \mathrm{C}$-domains to each other is quite low. However, additional interactions described above allow them to form a dimer and to keep this dimer at the central region of the molecule through the interaction in which FpA and FpB seem to be critical. ${ }^{21,43}$ Thus, removal of these fibrinopeptides upon fibrinogen to fibrin conversion disrupts interaction of the $\alpha \mathrm{C}$-domains with the central region and results in their dissociation from each other (-Fig. 6B). However, the monomeric state of the $\alpha \mathrm{C}$-domains is transient. Even with such a low affinity to each other, the $\alpha \mathrm{C}$-domains rapidly associate intermolecularly since the local concentration of the $\alpha \mathrm{C}$-domains in the fibrin polymer is very high. Such association involves interaction between their $\mathrm{N}$-terminal subdomains through the $\beta$ hairpin swapping mechanism (-Fig. 5A) and results in formation of $\alpha$ C polymers (-Fig. 6C-E). In addition, interaction of the C-terminal subdomains with the $\alpha \mathrm{C}$-connectors in polymeric fibrin (-Fig. 6D) may promote formation of $\alpha \mathrm{C}$ polymers. This interaction also provides proper positioning of the reactive Lys and Gln residues, which were previously localized in the $\mathrm{C}$-terminal subdomain and the $\alpha \mathrm{C}$-connector, respectively, ${ }^{28,53,54}$ to facilitate their covalent cross-linking by factor XIIIa. In agreement, factor XIIIa-mediated crosslinking of oligomers formed by the A $\alpha 221-610$ fragment was

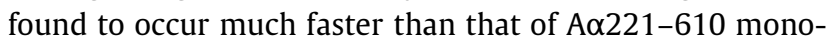
mers. ${ }^{41}$ Such interactions and cross-linking require an antiparallel arrangement of the $\alpha \mathrm{C}$ regions that are coming from the neighboring fibrin molecules, as shown schematically in - Fig. 6D.

It should be noted that formation of $\alpha C$ polymers requires fibrin polymerization through knob-hole interactions, as

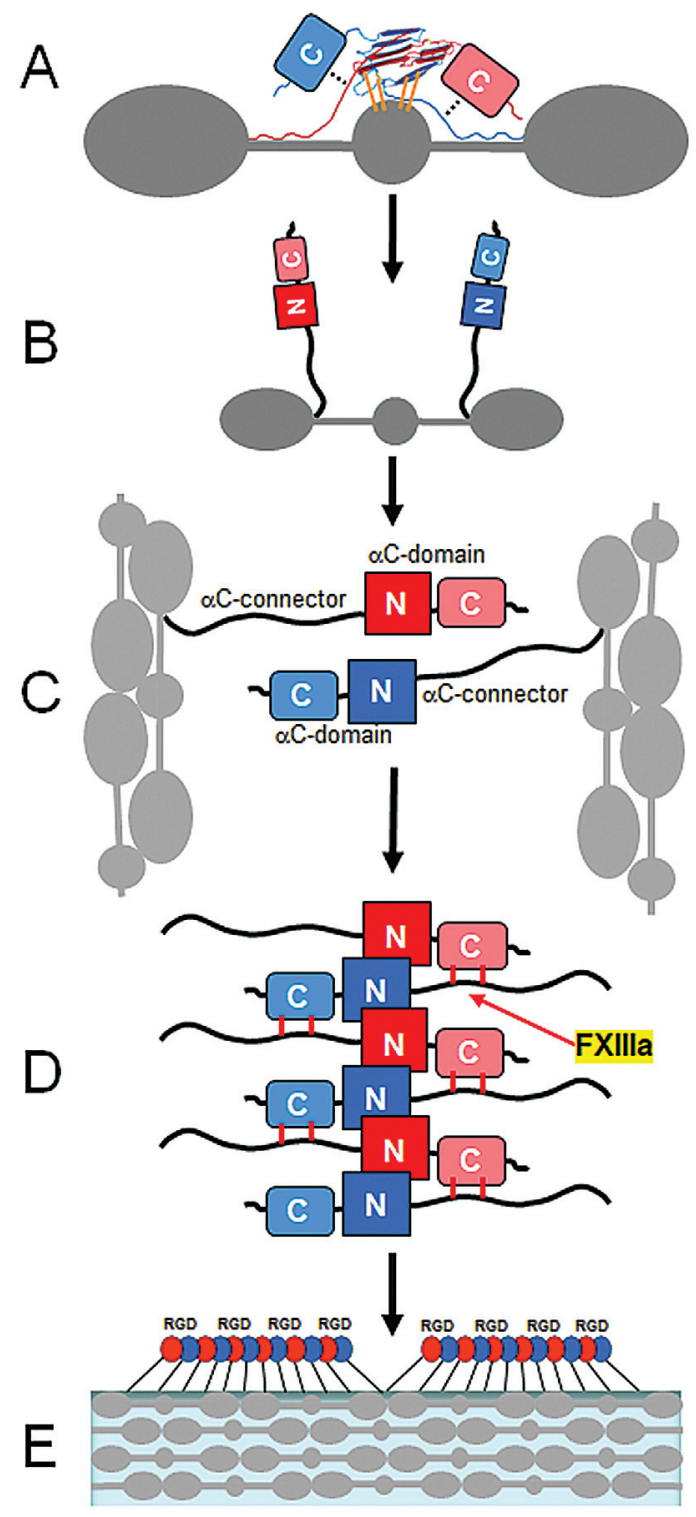

Fig. 6 Schematic representation of the $\alpha C$-domains in fibrinogen, fibrin monomers, and in fibrin $\alpha C$ polymers. Panel (A) shows how two $\alpha C$-domains may interact with each other in the fibrinogen molecule through the $\mathrm{N}$-terminal subdomains by the $\beta$-hairpin swapping mechanism presented in - Fig. 5A; the interaction between the Cterminal subdomains and the $\alpha C$-connectors is shown by dotted lines, fibrinopeptides $A$ and $B$ are shown by orange bars. The location shown for the $\mathrm{N}$ - and C-terminal subdomains is arbitrary. Panel (B) shows dissociation of the $\alpha C$-domains from each other and from the central part of the molecule in fibrin monomer. Panels (C) and (D) show interaction between the $\alpha \mathrm{C}$-domains upon fibrin polymerization resulting in $\alpha C$ polymer formation. Interaction between the $\mathrm{N}$-terminal subdomains may occur by the $\beta$-hairpin swapping mechanism presented in - Fig. 5A; the C-terminal subdomains interact with the $\alpha \mathrm{C}$-connectors and are cross-linked to the $\alpha \mathrm{C}$-connectors by factor XIIla (see text); the cross-linking is shown by two vertical red bars. Panel (E) represents schematically $\alpha C$ polymers in which the $\alpha C$ domains (blue and red balls) are attached to the surface of fibrin fibers through the $\alpha C$-connectors (black lines), whose length may restrict the length of the polymers (see text). Although the $\alpha \mathrm{C}$-polymers are shown only on the surface of a fibrin fiber, they may also be formed between protofibrils, as shown in panel (C), and inside the fiber. The location of the RGD integrin-binding motifs on $\alpha C$ polymers is also shown. 
mentioned above. In agreement, a recent study revealed that intermolecular interaction between the $\alpha \mathrm{C}$-domains alone is too weak to be able to lead to any fibrin(ogen) assembly in the absence of knob-hole engagement. ${ }^{55}$ It should also be noted that the size of $\alpha C$ polymers in cross-linked fibrin is restricted. Namely, analysis of the size distribution of solubilized $\alpha \mathrm{C}$ polymers from cross-linked human fibrin performed by dynamic light scattering revealed that the major fraction of such polymers includes species whose size varies from approximately 60 to 90 monomeric units, suggesting that these many $\alpha C$ regions may form linear $\alpha C$ polymers in cross-linked fibrin. ${ }^{47}$ The length of such polymers $(\approx 130-$ $200 \mathrm{~nm}$ ) is much shorter than that of individual fibrin fibers, thereby excluding the possibility that fiber length restricts the size of $\alpha C$ polymers in fibrin. Therefore, we suggested that their size may be restricted by the length of the $\alpha \mathrm{C}$ connectors that allows individual $\alpha C$-domains to move from the bulk of the molecules and cluster into polymers, ${ }^{47}$ as shown in - Fig. 6E. Analysis of cross-linked $\alpha C$ polymers from fibrins of different species, whose $\alpha C$-connectors' length significantly varies, could test this suggestion.

\section{Role of the aC Regions in Fibrin Polymerization}

The switch from intra- to intermolecular interactions between the $\alpha \mathrm{C}$-domains described above suggests that they should participate in fibrin assembly. Indeed, the involvement of the C-terminal portions of fibrinogen's A $\alpha$ chains in fibrin assembly process was reported more than five decades ago. ${ }^{56}$ Fibrinogen in the circulation is heterogeneous in size, mainly due to proteolytic truncation of the C-terminal portions of its A $\alpha$ chains. ${ }^{24}$ Experiments with different fractions of fibrinogen showed that lower molecular weight fibrinogen has longer clotting times and turbidity curves with longer lag period, which reflects protofibril formation, and slower turbidity rise. ${ }^{56}$ In agreement, experiments with fibrin monomer and monomeric fragment $\mathrm{X}_{1}$ devoid of the $\alpha \mathrm{C}$-domain(s) revealed that these domains accelerate formation of protofibrils and fibers. ${ }^{30}$ The fact that this effect is more pronounced in dilute solutions ${ }^{30}$ suggests that these domains may play a significant role in the early stages of fibrin assembly, at which the concentration of fibrin is low. Our subsequent study performed by EM and turbidity measurements confirmed that the $\alpha \mathrm{C}$-domain interactions affect the rate of fibrin assembly and revealed that they also affect clot structure. ${ }^{26}$ It should be noted that a fibrinogen hybrid variant, where the human $\alpha C$ regions were replaced with the homologous chicken region, has impaired lateral aggregation, such that fibrin monomers can assemble into protofibrils or protofibril-like structures, but these either cannot assemble into fibers or assemble into very thin fibers. ${ }^{57}$ Since chicken $\alpha C$ regions practically do not have $\alpha \mathrm{C}$-connectors, this observation suggests the important role of the $\alpha \mathrm{C}$ connectors in the fibrin assembly process. A recent study ${ }^{58}$ confirmed the importance of the $\alpha C$-connectors in fibrin assembly and revealed an additional role of the $\alpha C$ regions in this process. Namely, it was shown that the recombinant fibrinogen variant $\alpha 390$, truncated before the $\alpha C$-domain, produced a clot consisting of thinner fibers making up a denser structural network. In contrast, the $\alpha 220$ variant, truncated at the start of the $\alpha \mathrm{C}$-connector, produced clots that were porous with short and stunted fibers, suggesting that the $\alpha C$ regions are key for promoting longitudinal fiber growth.

To investigate the involvement of the $\alpha C$ regions in the early stages of fibrin polymerization, high-resolution atomic force microscopy was used to image fibrinogen, oligomers of fibrin, and protofibrils. ${ }^{59}$ Plasma-purified full-length human fibrinogen was compared with des- $\alpha \mathrm{C}$ fibrinogen lacking most of the $\alpha \mathrm{C}$ regions. In fibrinogen converted to fibrin by treatment with thrombin, up to $80 \%$ of the potentially existing $\alpha C$ regions were visualized and quantified. They were highly heterogeneous in both length and conformation. Conversion of fibrinogen to fibrin was accompanied by an increase in the incidence and length of the $\alpha \mathrm{C}$ regions as well as transitions from more compact conformations, such as a globule on a string, to longer and more flexible extensions. Concurrent dynamic turbidimetry, as well as confocal microscopy and scanning EM, revealed that trimming of the $\alpha C$ regions slowed down fibrin formation, which correlated with longer protofibrils, thinner fibers, and a denser fibrin network. No other structural differences, except for the incidence of appearance of the $\alpha C$ regions, were observed in the laterally aggregated protofibrils made of the full-length or des- $\alpha C$ fibrinogens, suggesting a purely kinetic effect of the $\alpha C$ regions on the fibrin architecture. In conclusion, these studies provide a molecular structural basis for a role of the $\alpha C$ regions in enhancing the early stages of fibrin polymerization.

\section{Role of the aC Regions in Mechanical Properties of Fibrin Clots}

The role of the $\alpha \mathrm{C}$ polymers in the mechanical properties of fibrin, underestimated in the past, has been studied intensively more recently. A recombinant fibrinogen, $\alpha 251$ with $\alpha$ chains truncated at residue 251 and devoid of its $\alpha C$-domains and most of the $\alpha \mathrm{C}$-connectors, is greatly impaired in lateral aggregation, with clots made up of thinner and denser fibers with more branch points, and permeability nearly half that of control clots. ${ }^{60,61}$ The $\alpha 251$ fibrin clots were much less stiff and showed more plastic deformation, indicating that interactions between the $\alpha \mathrm{C}$-domains in normal clots play a major role in determining the clot's viscoelastic properties. The stiffness of fibrin clots is substantially increased by interactions between $\alpha \mathrm{C}$ regions, and factor XIIIa-catalyzed covalent cross-linking of the $\alpha$ chains contributes further to increased fibrin clot stiffness. ${ }^{60}$ A recent study with fibrinogen variant $\alpha 390$, devoid of the $\alpha C$-domains, which revealed reduced mechanical stability of fibrin clots formed by this variant, ${ }^{58}$ further demonstrates the important contribution of the $\alpha \mathrm{C}$-domains to mechanical properties of fibrin clots.

Studies of the mechanical properties of single fibrin fibers by atomic force microscopy-based nanomanipulation have shown that cross-linked fibers are twofold stiffer than 
uncross-linked fibers and $\alpha C$ regions are responsible for the low modulus, large extensibility, and strain stiffening. ${ }^{62}$ Using a recombinant fibrinogen variant that is incapable of $\gamma$ chain cross-linking, it was shown that individual fibers with $\alpha$ chain cross-linking only are $2.5 \times$ stiffer and $1.5 \times$ more elastic than those without cross-linking. ${ }^{63}$ In contrast, single fibers with full cross-linking are $3.75 \times$ stiffer, $1.2 \times$ more elastic, and $1.2 \times$ less extensible. Similar studies with the same methodology combined with high-frame-rate imaging used to measure the recoil dynamics of individual fibrin fibers found that the recoil was orders of magnitude faster than anticipated from models involving protein refolding. ${ }^{64}$ Molecular-dynamics simulations point to the unstructured $\alpha \mathrm{C}$ regions as being responsible for the elastic recoil of the fibers. Based on a combination of several studies of the $\alpha C$ regions in fibrin mechanics, a structural model of fibrinogen, including its folded core, $\alpha \mathrm{C}$ connector, and $\alpha \mathrm{C}$-domain, has been proposed for the stress-strain behavior of individual fibrin fibers. ${ }^{65}$

\section{Conversion of Fibrinogen into Fibrin Results in the Exposure of Multiple Binding Sites in Its aC-Domains}

Numerous data indicate that fibrinogen is inert in the circulation but becomes highly reactive after conversion to fibrin. Likewise, the $\alpha \mathrm{C}$-domains are inert in the fibrinogen molecule but become highly reactive in fibrin. This, most probably, is connected with conformational changes upon formation of $\alpha$ polymers by the $\alpha \mathrm{C}$ regions and exposure of their numerous binding sites. For example, it was shown that the $\alpha C$-domains contain cryptic fibronectin-binding sites that are not accessible in fibrinogen but become exposed in fibrin. ${ }^{66,67}$ Similarly, the $\alpha C$-domains contain cryptic apolipoprotein(a)-binding sites that became available for interaction with apolipoprotein(a) only after conversion of fibrinogen into fibrin. ${ }^{68}$ Further, fibrinogen $\alpha C$-domains contain binding sites for plasminogen and tissue plasminogen activator ( $\mathrm{TPA}$ ) that are cryptic in fibrinogen and become accessible in fibrin to promote activation of plasminogen by tPA upon initiation of fibrin-dependent fibrinolysis. ${ }^{41,69-71}$ Interestingly, these binding sites are also nonreactive in the isolated $\alpha C$ regions; however, they become active in the $\alpha C$ polymers. ${ }^{41}$ Furthermore, the fibrinogen $\alpha \mathrm{C}$-domains contain cryptic binding sites for the plasmin inhibitor, $\alpha_{2}$-antiplasmin, that become exposed in fibrin ${ }^{72}$ and play an important role in the regulation of fibrinolysis. ${ }^{73}$

The $\alpha C$-domains also contain the RGD recognition motif (Arg572-Gly573-Asp574 residues of the C-terminal subdomain) that is known to interact with different integrin adhesion receptors. Although this sequence may not be involved in platelet aggregation through the interaction of fibrinogen with platelet integrin $\alpha$ IIb $\beta 3$, it is implicated in integrin interactions with fibrin. ${ }^{74,75}$ Interestingly, in fibrin, in which the $\alpha \mathrm{C}$-domains form $\alpha \mathrm{C}$ polymers, these integrinbinding motifs are clustered and juxtaposed in a highly ordered manner, forming some kind of linear tracks (-Fig. 6E). Such clustering was shown to increase their
RGD-dependent interaction with endothelial integrins $\alpha \mathrm{V} \beta 3$ and $\alpha \mathrm{V} \beta 5{ }^{76}$ The clustering also promotes endothelial cell migration and proliferation, ${ }^{77}$ the events that play an important role in wound healing. It should be noted that all above-mentioned interactions have been documented in in vitro experiments. Whether they play a role in various (patho)physiological conditions in vivo remains to be addressed.

\section{Conclusion}

The $\alpha \mathrm{C}$ regions were the most enigmatic structures in the fibrinogen molecule. For a long time, they were considered to be unordered ${ }^{20}$ until it was shown that they contain ordered structures and form two compact $\alpha \mathrm{C}$-domains attached to the bulk of the molecule by the flexible $\alpha \mathrm{C}$ connectors. ${ }^{10,11,23}$ Subsequent studies performed mainly in our laboratories revealed that in fibrinogen the $\alpha \mathrm{C}$-domains interact intramolecularly with each other and with the central region of the molecule. ${ }^{21,26,30,43}$ Further, upon conversion of fibrinogen into fibrin, the $\alpha \mathrm{C}$-domains dissociate from each other and from the central region of the molecule and re-associate in a new manner, intermolecularly, i.e., they switch from intra- to intermolecular interactions. ${ }^{1,30}$ The molecular mechanism of such a switch has been clarified ${ }^{47}$ and is summarized in the present review (-Fig. 6). The switch of the $\alpha$ C-domains to intermolecular interactions results in formation of $\alpha \mathrm{C}$ polymers in fibrin fibers in which the $\alpha \mathrm{C}$-domains interact with each other through their N-terminal subdomains, most probably by a domain swapping mechanism, and with the $\alpha C$-connectors. $^{38,47}$ Such interactions accelerate the fibrin assembly process, promote longitudinal fiber growth, and increase the mechanical stability of fibrin. ${ }^{30,58,60,61}$ Furthermore, formation of $\alpha \mathrm{C}$ polymers in fibrin results in the exposure and/or formation of their multiple binding sites ${ }^{41,66-68,70,72}$ that provide interactions of the $\alpha \mathrm{C}$ regions with different proteins and cell types and their participation in various physiological and pathological processes. Thus, these previously enigmatic parts of the fibrinogen molecule are finally revealing their true nature.

\section{Funding}

This work was supported by National Institutes of Health grants HL056051 to L.M., and HL030954, HL148227, and P01-HL40387 to J.W.W.

Conflict of Interest

None declared.

\section{References}

1 Weisel JW, Medved L. The structure and function of the $\alpha \mathrm{C}$ domains of fibrinogen. Ann N Y Acad Sci 2001;936:312-327

2 Soria J, Mirshahi S, Mirshahi SQ, et al. Fibrinogen $\alpha C$ domain: its importance in physiopathology. Res Pract Thromb Haemost 2019; 3(02):173-183

3 Medved L, Weisel JWFibrinogen and Factor XIII Subcommittee of Scientific Standardization Committee of International Society on 
Thrombosis and Haemostasis. Recommendations for nomenclature on fibrinogen and fibrin. J Thromb Haemost 2009;7(02): 355-359

4 Hall CE, Slayter HS. The fibrinogen molecule: its size, shape, and mode of polymerization.J Biophys Biochem Cytol 1959;5(01):11-16

5 Mosesson MW, Hainfeld J, Wall J, Haschemeyer RH. Identification and mass analysis of human fibrinogen molecules and their domains by scanning transmission electron microscopy. J Mol Biol 1981;153(03):695-718

6 Slayter HS. Electron microscopic studies of fibrinogen structure: historical perspectives and recent experiments. Ann N Y Acad Sci 1983;408:131-145

7 Williams RC. Morphology of fibrinogen monomers and of fibrin protofibrils. Ann N Y Acad Sci 1983;408:180-193

8 Erickson HP, Fowler WE. Electron microscopy of fibrinogen, its plasmic fragments and small polymers. Ann N Y Acad Sci 1983; 408:146-163

9 Weisel JW, Stauffacher CV, Bullitt E, Cohen C. A model for fibrinogen: domains and sequence. Science 1985;230(4732):1388-1391

10 Privalov PL, Medved LV. Domains in the fibrinogen molecule.J Mol Biol 1982;159(04):665-683

11 Medved LV, Gorkun OV, Privalov PL. Structural organization of Cterminal parts of fibrinogen A $\alpha$-chains. FEBS Lett 1983;160(12):291-295

12 Medved L, Litvinovich S, Ugarova T, Matsuka Y, Ingham K. Domain structure and functional activity of the recombinant human fibrinogen $\gamma$-module ( $\gamma 148-411)$. Biochemistry 1997;36(15): 4685-4693

13 Spraggon G, Everse SJ, Doolittle RF. Crystal structures of fragment D from human fibrinogen and its crosslinked counterpart from fibrin. Nature 1997;389(6650):455-462

14 Madrazo J, Brown JH, Litvinovich S, et al. Crystal structure of the central region of bovine fibrinogen (E5 fragment) at 1.4-A resolution. Proc Natl Acad Sci U S A 2001;98(21):11967-11972

15 Pechik I, Madrazo J, Mosesson MW, Hernandez I, Gilliland GL, Medved L. Crystal structure of the complex between thrombin and the central "E" region of fibrin. Proc Natl Acad Sci U S A 2004; 101(09):2718-2723

16 Yang Z, Mochalkin I, Veerapandian L, Riley M, Doolittle RF. Crystal structure of native chicken fibrinogen at 5.5-A resolution. Proc Natl Acad Sci U S A 2000;97(08):3907-3912

17 Yang Z, Kollman JM, Pandi L, Doolittle RF. Crystal structure of native chicken fibrinogen at 2.7 A resolution. Biochemistry 2001; 40(42):12515-12523

18 Brown JH, Volkmann N, Jun G, Henschen-Edman AH, Cohen C. The crystal structure of modified bovine fibrinogen. Proc Natl Acad Sci U S A 2000;97(01):85-90

19 Kollman JM, Pandi L, Sawaya MR, Riley M, Doolittle RF. Crystal structure of human fibrinogen. Biochemistry 2009;48(18): 3877-3886

20 Doolittle RF. Structural aspects of the fibrinogen to fibrin conversion. Adv Protein Chem 1973;27:1-109

21 Veklich YI, Gorkun OV, Medved LV, Nieuwenhuizen W, Weisel JW. Carboxyl-terminal portions of the $\alpha$ chains of fibrinogen and fibrin. Localization by electron microscopy and the effects of isolated $\alpha$ C fragments on polymerization. J Biol Chem 1993; 268(18):13577-13585

22 Procyk R, Medved L, Engelke KJ, Kudryk B, Blombäck B. Nonclottable fibrin obtained from partially reduced fibrinogen: characterization and tissue plasminogen activator stimulation. Biochemistry 1992;31(08):2273-2278

23 Tsurupa G, Tsonev L, Medved L. Structural organization of the fibrin(ogen) $\alpha$ C-domain. Biochemistry 2002;41(20):6449-6459

24 Mosesson MW. Fibrinogen heterogeneity. Ann N Y Acad Sci 1983; 408:97-113

25 Henschen A, McDonagh J. Fibrinogen, fibrin and factor XIII. In: Zwaal RFA, Hemker HC, eds. Blood Coagulation. Amsterdam: Elsevier Science Publishers; 1986:171-241
26 Gorkun OV, Veklich YI, Medved LV, Henschen AH, Weisel JW. Role of the $\alpha \mathrm{C}$ domains of fibrin in clot formation. Biochemistry 1994; 33(22):6986-6997

27 Rudchenko S, Trakht I, Sobel JH. Comparative structural and functional features of the human fibrinogen $\alpha \mathrm{C}$ domain and the isolated $\alpha \mathrm{C}$ fragment. Characterization using monoclonal antibodies to defined $\mathrm{COOH}$-terminal A $\alpha$ chain regions. J Biol Chem 1996;271(05):2523-2530

28 Matsuka YV, Medved LV, Migliorini MM, Ingham KC. Factor XIIIacatalyzed cross-linking of recombinant $\alpha \mathrm{C}$ fragments of human fibrinogen. Biochemistry 1996;35(18):5810-5816

29 Protopopova AD, Barinov NA, Zavyalova EG, Kopylov AM, Sergienko VI, Klinov DV. Visualization of fibrinogen $\alpha \mathrm{C}$ regions and their arrangement during fibrin network formation by highresolution AFM. J Thromb Haemost 2015;13(04):570-579

30 Medved' LV, Gorkun OV, Manyakov VF, Belitser VA. The role of fibrinogen $\alpha$ C-domains in the fibrin assembly process. FEBS Lett 1985;181(01):109-112

31 Murakawa M, Okamura T, Kamura T, Shibuya T, Harada M, Niho Y. Diversity of primary structures of the carboxy-terminal regions of mammalian fibrinogen $\mathrm{A} \alpha$-chains. Characterization of the partial nucleotide and deduced amino acid sequences in five mammalian species; rhesus monkey, pig, dog, mouse and Syrian hamster. Thromb Haemost 1993;69(04):351-360

32 Williamson MP. The structure and function of proline-rich regions in proteins. Biochem J 1994;297(Pt 2):249-260

33 Makarov AA, Lobachov VM, Adzhubei IA, Esipova NG. Natural polypeptides in left-handed helical conformation. A circular dichroism study of the linker histones' C-terminal fragments and $\beta$-endorphin. FEBS Lett 1992;306(01):63-65

34 Sreerama N, Woody RW. Poly(pro)II helices in globular proteins: identification and circular dichroic analysis. Biochemistry 1994; 33(33):10022-10025

35 Lugovskoy EV, Gritsenko PG, Kolesnikova IN, Lugovskaya NE, Komisarenko SV. A neoantigenic determinant in coiled coil region of human fibrin $\beta$-chain. Thromb Res 2009;123(05): 765-770

36 Doolittle RF. Structural basis of the fibrinogen-fibrin transformation: contributions from X-ray crystallography. Blood Rev 2003; 17(01):33-41

37 Doolittle RF, Kollman JM. Natively unfolded regions of the vertebrate fibrinogen molecule. Proteins 2006;63(02):391-397

38 Burton RA, Tsurupa G, Medved L, Tjandra N. Identification of an ordered compact structure within the recombinant bovine fibrinogen alphaC-domain fragment by NMR. Biochemistry 2006;45 (07):2257-2266

39 Burton RA, Tsurupa G, Hantgan RR, Tjandra N, Medved L. NMR solution structure, stability, and interaction of the recombinant bovine fibrinogen alphaC-domain fragment. Biochemistry 2007; 46(29):8550-8560

40 Tsurupa G, Hantgan RR, Burton RA, Pechik I, Tjandra N, Medved L. Structure, stability, and interaction of the fibrin(ogen) alphaCdomains. Biochemistry 2009;48(51):12191-12201

41 Tsurupa G, Mahid A, Veklich Y, Weisel JW, Medved L. Structure, stability, and interaction of fibrin $\alpha C$-domain polymers. Biochemistry 2011;50(37):8028-8037

42 Tsurupa G, Veklich Y, Hantgan R, Belkin AM, Weisel JW, Medved L. Do the isolated fibrinogen alphaC-domains form ordered oligomers? Biophys Chem 2004;112(2-3):257-266

43 Litvinov RI, Yakovlev S, Tsurupa G, Gorkun OV, Medved L, Weisel JW. Direct evidence for specific interactions of the fibrinogen alphaC-domains with the central $\mathrm{E}$ region and with each other. Biochemistry 2007;46(31):9133-9142

44 McKee PA, Mattock P, Hill RL. Subunit structure of human fibrinogen, soluble fibrin, and cross-linked insoluble fibrin. Proc Natl Acad Sci U S A 1970;66(03):738-744

45 Schwartz ML, Pizzo SV, Sullivan JB, Hill RL, McKee PA. A comparative study of crosslinked and noncrosslinked fibrin from the major 
classes of vertebrates. Thromb Diath Haemorrh 1973;29(02): 313-338

46 Fretto LJ, McKee PA. Structure of $\alpha$-polymer from in vitro and in vivo highly cross-linked human fibrin. J Biol Chem 1978;253(18): 6614-6622

47 Tsurupa G, Pechik I, Litvinov RI, et al. On the mechanism of $\alpha \mathrm{C}$ polymer formation in fibrin. Biochemistry 2012;51(12): 2526-2538

48 Zhang C, Kim SH. The anatomy of protein $\beta$-sheet topology. J Mol Biol 2000;299(04):1075-1089

49 Bennett MJ, Schlunegger MP, Eisenberg D. 3D domain swapping: a mechanism for oligomer assembly. Protein Sci 1995;4(12): 2455-2468

50 Jaskólski M. 3D domain swapping, protein oligomerization, and amyloid formation. Acta Biochim Pol 2001;48(04):807-827

51 Liu Y, Eisenberg D. 3D domain swapping: as domains continue to swap. Protein Sci 2002;11(06):1285-1299

52 Bennett MJ, Eisenberg D. The evolving role of 3D domain swapping in proteins. Structure 2004;12(08):1339-1341

53 Cottrell BA, Strong DD, Watt KW, Doolittle RF. Amino acid sequence studies on the $\alpha$ chain of human fibrinogen. Exact location of cross-linking acceptor sites. Biochemistry 1979;18 (24):5405-5410

54 Corcoran DH, Ferguson EW, Fretto LJ, McKee PA. Localization of a cross-link donor site in the $\alpha$-chain of human fibrin. Thromb Res 1980;19(06):883-888

55 Duval C, Profumo A, Aprile A, et al. Fibrinogen $\alpha$ C-regions are not directly involved in fibrin polymerization as evidenced by a "Double-Detroit" recombinant fibrinogen mutant and knobsmimic peptides. J Thromb Haemost 2020;18(04):802-814

56 Mosesson MW, Alkjaersig N, Sweet B, Sherry S. Human fibrinogen of relatively high solubility. Comparative biophysical, biochemical, and biological studies with fibrinogen of lower solubility. Biochemistry 1967;6(10):3279-3287

57 Ping L, Huang L, Cardinali B, Profumo A, Gorkun OV, Lord ST. Substitution of the human $\alpha C$ region with the analogous chicken domain generates a fibrinogen with severely impaired lateral aggregation: fibrin monomers assemble into protofibrils but protofibrils do not assemble into fibers. Biochemistry 2011;50 (42):9066-9075

58 McPherson HR, Duval C, Baker SR, et al. Fibrinogen $\alpha C$-subregions critically contribute blood clot fibre growth, mechanical stability, and resistance to fibrinolysis. eLife 2021;10:e68761

59 Protopopova AD, Litvinov RI, Galanakis DK, et al. Morphometric characterization of fibrinogen's $\alpha C$ regions and their role in fibrin self-assembly and molecular organization. Nanoscale 2017;9 (36):13707-13716

60 Collet J-P, Veklich Y, MullinJL, Gorkun OV, Lord ST, Weisel JW. The $\alpha C$ domains of fibrinogen affect the structure of the clot and its physical and biochemical properties. Thromb Haemost 1999;82:692

61 Collet J-P, Moen JL, Veklich YI, et al. The alphaC domains of fibrinogen affect the structure of the fibrin clot, its physical properties, and its susceptibility to fibrinolysis. Blood 2005;106 (12):3824-3830
62 Houser JR, Hudson NE, Ping L, et al. Evidence that $\alpha \mathrm{C}$ region is origin of low modulus, high extensibility, and strain stiffening in fibrin fibers. Biophys J 2010;99(09):3038-3047

63 Helms CC, Ariëns RA, Uitte de Willige S, Standeven KF, Guthold M. $\alpha-\alpha$ Cross-links increase fibrin fiber elasticity and stiffness. Biophys J 2012;102(01):168-175

64 Hudson NE, Ding F, Bucay I, et al. Submillisecond elastic recoil reveals molecular origins of fibrin fiber mechanics. Biophys $\mathrm{J}$ 2013;104(12):2671-2680

65 Averett RD, Menn B, Lee EH, Helms CC, Barker T, Guthold M. A modular fibrinogen model that captures the stress-strain behavior of fibrin fibers. Biophys J 2012;103(07):1537-1544

66 Makogonenko E, Tsurupa G, Ingham K, Medved L. Interaction of fibrin(ogen) with fibronectin: further characterization and localization of the fibronectin-binding site. Biochemistry 2002;41 (25):7907-7913

67 Makogonenko E, Ingham KC, Medved L. Interaction of the fibronectin $\mathrm{COOH}$-terminal Fib-2 regions with fibrin: further characterization and localization of the Fib-2-binding sites. Biochemistry 2007;46(18):5418-5426

68 Tsurupa G, Ho-Tin-Noe B, Loyau S, Angles-Cano E, Medved L. Identification and characterization of novel Lys-independent apo (a)-binding sites within fibrinogen $\alpha C$-domains. J Biol Chem 2003; 278(39):37154-37159

69 Tsurupa G, Medved L. Identification and characterization of novel tPA- and plasminogen-binding sites within fibrin(ogen) $\alpha \mathrm{C}$ domains. Biochemistry 2001;40(03):801-808

70 Tsurupa G, Medved L. Fibrinogen $\alpha \mathrm{C}$ domains contain cryptic plasminogen and tPA binding sites. Ann N Y Acad Sci 2001; 936:328-330

71 Medved L, Nieuwenhuizen W. Molecular mechanisms of initiation of fibrinolysis by fibrin. Thromb Haemost 2003;89(03): 409-419

72 Tsurupa G, Yakovlev S, McKee P, Medved L. Noncovalent interaction of $\alpha(2)$-antiplasmin with fibrin(ogen): localization of $\alpha(2)$ antiplasmin-binding sites. Biochemistry 2010;49(35): 7643-7651

73 Fraser SR, Booth NA, Mutch NJ. The antifibrinolytic function of factor XIII is exclusively expressed through $\alpha_{2}$-antiplasmin crosslinking. Blood 2011;117(23):6371-6374

74 Litvinov RI, Farrell DH, Weisel JW, Bennett JS. The platelet integrin $\alpha$ IIb $\beta 3$ differentially interacts with fibrin versus fibrinogen. J Biol Chem 2016;291(15):7858-7867

75 Höök P, Litvinov RI, Kim OV, et al. Strong binding of the platelet integrin $\alpha$ IIb $\beta 3$ to fibrin clots: a potential target to destabilize thrombi. Sci Rep 2017;7(01):13001

76 Belkin AM, Tsurupa G, Zemskov E, Veklich Y, Weisel JW, Medved L. Transglutaminase-mediated oligomerization of the fibrin(ogen) alphaC domains promotes integrin-dependent cell adhesion and signaling. Blood 2005;105(09):3561-3568

77 Yakovlev S, Mikhailenko I, Tsurupa G, Belkin AM, Medved L. Polymerisation of fibrin $\alpha \mathrm{C}$-domains promotes endothelial cell migration and proliferation. Thromb Haemost 2014;112(06): 1244-1251 